\title{
Characterizing Early Maternal Style in a Population of Guide Dogs
}

\author{
Emily E. Bray ${ }^{1 *}$, Mary D. Sammel ${ }^{2}$, Dorothy L. Cheney ${ }^{3}$, James A. Serpell ${ }^{4}$ and \\ Robert M. Seyfarth ${ }^{1}$
}

${ }^{1}$ Department of Psychology, University of Pennsylvania, Philadelphia, PA, USA, ${ }^{2}$ Department of Biostatistics and Epidemiology, Perelman School of Medicine, University of Pennsylvania, Philadelphia, PA, USA, ${ }^{3}$ Department of Biology, University of Pennsylvania, Philadelphia, PA, USA, ${ }^{4}$ Department of Clinical Studies, School of Veterinary Medicine, University of Pennsylvania, Philadelphia, PA, USA

OPEN ACCESS

Edited by: Thomas Bugnyar, University of Vienna, Austria

Reviewed by:

Friederike Range, University of Vienna, Austria

Sonja Elena Koski,

University of Helsinki, Finland

*Correspondence:

Emily E. Bray

ebray@sas.upenn.edu

Specialty section:

This article was submitted to

Comparative Psychology,

a section of the journal

Frontiers in Psychology

Received: 12 November 2016

Accepted: 25 January 2017

Published: 10 February 2017

Citation:

Bray EE, Sammel MD, Cheney DL, Serpell JA and Seyfarth RM (2017) Characterizing Early Maternal Style in

a Population of Guide Dogs.

Front. Psychol. 8:175

doi: 10.3389/fpsyg.2017.00175
In both humans and non-humans, differences in maternal style during the first few weeks of life can be reliably characterized, and these differences affect offspring's temperament and cognition in later life. Drawing on the breeding population of dogs at The Seeing Eye, a guide dog school in Morristown, New Jersey, we conducted videotaped focal follows on 21 mothers and their litters ( $n=138$ puppies) over the first 3 weeks of the puppies' lives in an effort to characterize maternal style. We found that a mother's attitude and actions toward her offspring varied naturally between individuals, and that these variations could be summarized by a single principal component, which we described as Maternal behavior. This component was stable across weeks, associated with breed, litter size, and parity, but not redundant with these attributes. Furthermore, this component was significantly associated with an independent experimental measure of maternal behavior, and with maternal stress as measured by salivary cortisol. In summary, Maternal behavior captured a significant proportion of the variation in maternal style; was stable over time; and had both discriminant and predictive validity.

Keywords: maternal style, canine, guide dogs, nursing, licking/grooming, behavior

\section{INTRODUCTION}

In rodents and primates, early separation from the mother has been shown to play a negative role in offspring's later expression of emotions, as measured by task performance and glucocorticoid receptor density (e.g., Aisa et al., 2008); later behavior, as measured by fear conditioning (e.g., Kosten et al., 2006); and later cognition, as measured by performance in inhibitory control tasks (e.g., Pryce et al., 2004). However, simply having a mother present is not enough; her behavior toward her offspring also matters. Individual differences in maternal styles are both distinguishable and important for later outcomes. For example, in Old World monkeys, mothers naturally fall along separate scales of infant rejection (tolerance of contact, carrying, and nursing) and protection (levels of grooming, proximity, and tendency to limit offspring exploration; e.g., Fairbanks, 1996; Parker and Maestripieri, 2011). In rodents, mothers vary in the amount of licking-grooming (LG) and arched-back nursing (ABN) that they display toward their offspring (e.g., Liu et al., 2000; Meaney, 2001; Fish et al., 2004).

Importantly, these variations in maternal style have real biological and cognitive consequences for the offspring. Rhesus macaque offspring who face repeated rejection by their mothers have lower levels of serotonin later in life (Parker and Maestripieri, 2011). Rodent pups raised by high LG-ABN mothers show better spatial memory (Liu et al., 2000) and exhibit muted stress 
responses when compared to offspring raised by low LG-ABN mothers (Francis et al., 1999). Researchers have implicated neural mechanisms through which high levels of early maternal care, and specifically tactile stimulation, might lead to superior spatial cognition and altered stress responses. Prominently featured in most theories is the hippocampus, which is widely acknowledged to play a role in spatial memory (e.g., Clayton and Krebs, 1994). High levels of early stimulation by the mother are thought to lead to increased NMDA receptors, which in turn facilitate the release of brain-derived neurotrophic factor and hippocampal synaptogenesis (e.g., Meaney, 2001; Fish et al., 2004). Liu et al. (2000) found significantly more NMDA receptors in the rat offspring of high LG-ABN mothers as early as 8 days and continuing into adulthood. Thus, it would appear that, above and beyond the presence of a mother figure during early development, the content and quality of interaction that the mother provides is extremely important for later outcomes.

Despite their close association with humans, comparatively little is known about the effects of maternal style on the cognitive and emotional development of domestic dogs (Czerwinski et al., 2016b; Serpell et al., 2016). In dogs, mothers provide care for at least the first 5 weeks postpartum and puppies reach sexual maturity in roughly 6-12 months (Morey, 1994). This relatively abbreviated life history makes dogs an ideal candidate in which to study the effects of maternal care. And yet surprisingly little progress has been made in characterizing early maternal style or documenting its effects on later offspring behavior, temperament, and outcomes. Questionnaire studies reveal that dog breeders attach little to no importance to maternal care when considering breeding stock (Leroy et al., 2007; Czerwinski et al., 2016a). However, as results from the broader animal literature indicate that maternal care is extremely important, understanding its effects in dogs specifically is crucial. Given that many working dog organizations breed their own animals in highly controlled and supervised environments, determining the types of maternal behaviors that lead to favorable outcomes and then encouraging and selecting for those maternal styles could be a highly effective strategy for increasing program graduation rates.

The few studies that have examined maternal care in dogs are consistent with the results from other species. For example, when puppies weaned at 4-6 weeks were compared with those weaned at 8-12 weeks, puppies weaned later were less prone to behavioral problems at older ages (Fält and Wilsson, 1979; Pierantoni and Verga, 2007; Pierantoni et al., 2011), reinforcing the view that maternal separation can have negative effects on behavior. Similarly, using a questionnaire, Tiira and Lohi (2015) had owners rate mothers on a scale of one (low levels of maternal interest) to seven (high levels of interest), and found that lower levels of maternal care were linked to fear and anxiety in offspring as adults.

In two recent studies, investigators monitored differences in mothers' natural interactions with their puppies over the first 3 weeks of life. Foyer et al. (2016) monitored motherpup interactions of German Shepherd dogs bred to be military working dogs, using five variables associated with the time that mothers spent in contact and interacting with their puppies. These variables loaded onto one principal component, which was associated with pup temperament at up to 18 months of age. In a similar study of beagles, Guardini et al. (2016) used four variables to assess mother-pup behavior during a daily 15-min period for the first 3 weeks of life. Again, these variables reduced into a single principal component reflecting amount of maternal care, which was significantly correlated with measures of puppy temperament at 2 months.

Our goal in the current study was to categorize the variations in maternal behavior during the first 3 weeks after birth and to use independent concurrent measures of behavior and hormones to validate our observations. We hypothesized that if there are consistent behavioral differences among mothers' interactions with their puppies, these differences might be associated both with maternal behavior in other contexts and with maternal cortisol levels. This method of validating a behavioral profile with behaviors and physiological factors that were not considered when building the profile has been used previously in studies of primates (Seyfarth et al., 2012).

Subjects were mothers and puppies bred to be guide dogs for the blind and visually impaired. We chose this population for several reasons. First, they provided us with a large sample of subjects that were housed and reared under controlled conditions, allowing for systematic observations. Cooperation of The Seeing Eye ${ }^{\circledR}$ personnel gave us access to background data on breed, age, and parity, allowed uninterrupted access to the same dogs across a variety of ages, and permitted us to conduct targeted experiments and standardized hormonal measurements. Finally, by around 16 months of age, the puppies in our study entered training as Seeing Eye ${ }^{\circledR}$ dogs, a process that led to either success or failure in the program. The problemsolving skills demanded of guide dogs are extremely complex, with exacting temperamental as well as cognitive requirements (Johnston, 1990). Guide dogs need to be sufficiently motivated to learn and tackle tasks, but also calm enough to rest quietly for hours at a time while their handlers are at work. Furthermore, they must filter the sensory information they encounter, giving full attention to relevant material (e.g., the flow of traffic) while ignoring the rest (e.g., passersby attempting to pet or play). They also need to differentiate among subtle commands (e.g., "wait" [short period of time] vs. "stay" [long period of time]); appropriately navigate environmental stimuli (e.g., escalators, revolving doors, curbs); resist temptation (e.g., dropped food on a restaurant floor); and respond to unanticipated events (e.g., an open manhole in the sidewalk, a closure along the usual route). The long-term goal of our research, therefore, was to examine possible associations between maternal behavior, measures of temperament and cognition in young adult dogs, and ultimate success in the Seeing Eye ${ }^{\circledR}$ program. The present paper constitutes a first step, by characterizing variation in maternal style. In upcoming papers (Bray et al., unpublished data), we examine the relationship between early maternal style and offspring performance on tests of temperament and cognition around 16 months, as well as offspring's outcome in the Seeing Eye ${ }^{\circledR}$ program.

In Part 1 of the study, we monitored maternal interactions in 21 litters that included three different breeds. We found that variation in seven behavioral measures could be summarized 
by one principal component, called Maternal behavior. Because breed, litter size, birth season, parity, and litter sex ratio have been identified in previous studies as potentially affecting both maternal behavior (Guardini et al., 2015; Foyer et al., 2016) and/or later puppy behavior (e.g., Borchelt, 1983; Wilsson and Sundgren, 1998; van der Waaij et al., 2008; Foyer et al., 2013, 2016), we then tested for associations between these variables and maternal style.

In Part 2, we validated our measure of maternal style by testing its association with experimental measures of maternal preference for puppies over a human visitor.

Finally, in Part 3, we searched for associations between maternal style and maternal stress levels before and after a brief separation from their puppies. By describing observed maternal style and ensuring it was related to other measures within the same timeframe, we aim to generate data that will provide the starting point for explicit predictions in subsequent work.

\section{GENERAL METHODS}

Mothers and puppies were housed at the breeding facility at The Seeing Eye, Inc. (Morristown, NJ, USA), a philanthropic organization that breeds, raises, and trains guide dogs for the blind and visually impaired. Mothers and puppies were the property of The Seeing Eye, Inc., which granted informed consent to all aspects of the study. All testing procedures adhered to regulations set forth and approved by the University of Pennsylvania Institutional Animal Care and Use Committee (Protocol \#805210).

The mothers in the study had all spent the first year and a half of their life with volunteer puppy-raisers where they received extensive socialization. During this time, the dogs were provided with basic obedience training, taken on field trips, and exposed to as many environments, people, and objects as possible. Dogs chosen to be breeders then completed 2 months of guide dog training and were handpicked to enter the breeding program based on their health, behavior, and genetic diversity.

At the breeding station, adult dogs were walked daily by volunteers and assigned to a staff member who continued to train them in basic obedience and agility. They were housed socially with one to two kennelmates in two adjoining $9^{\prime}$ by $10^{\prime}$ indoor pens, with access to two adjoining $9^{\prime}$ by $12^{\prime}$ outside pens. Pens were arranged in a circle so that dogs had visual access to all other pens and all humans that entered the room. About 1 week prior to their whelping date, mothers were transferred from the breeding wing to the whelping wing of the facility. Housing conditions here were similar to those in the breeding station, except that mothers were now housed singly. The inside pen was equipped with a round hard plastic kiddie pool ( $6^{\prime}$ diameter $\times 1^{\prime}$ tall) lined with towels, which served as the whelping pool. Post-birth, puppies remained in the whelping pool for the first 3 weeks. While training was discontinued for the 6 weeks that mothers spent in the whelping wing, mothers still received daily exercise and attention from volunteers and staff. Mothers remained in the whelping wing until their puppies were weaned at 5 weeks postpartum, at which point they returned to the breeding wing of the facility. Mothers received food 3 times a day and water was always available. The lights were switched on around 6:30 and turned off at 23:00 .

Over the study period (February-August 2014), 23 mothers whelped at the breeding station. Two of these mothers were excluded from the study due to atypical circumstances: one for having only one puppy and one for being separated from her litter over long periods prior to weaning. Thus, our final sample consisted of 21 mothers (nine German Shepherds, eight Labrador Retrievers, and four Golden Retriever; see Table 1) and their litters ( $n=138$ puppies).

\section{PART 1: OBSERVATIONS OF MATERNAL STYLE}

\section{Methods}

During the post-whelping period when mothers were in the same pen with their litters, our goal was to characterize maternal style by measuring variation in behavior across mothers. We aimed to create a maternal style profile of each mother through intensive video observations, allowing for later comparison of these results to experimental and endocrine measures collected in parallel.

\section{Subjects and Procedure}

We monitored each of the 21 mothers' behavior for 3 weeks (days 1-21) after birth. Three days per week, we videotaped two 10min sessions in the morning (between 9:00 and 12:00) and two 10-min sessions in the afternoon (between 15:00 and 18:00). We used Sony video cameras (HDR-PJ230, HDR-CX405) mounted on tripods outside of individual pens. Video footage was stored on hard drives and later analyzed using Datavyu (DatavyuTeam, 2014). We only recorded videos when the mother and all of her puppies were present in the pen, all humans were absent from the pen, and the mother was not eating. We coded a total of 6890 min of footage. Because access to monitoring was sometimes temporarily suspended due to cleaning, kennel staff, volunteer presence, or unforeseen circumstances (i.e., mothers whelping), the total video footage per litter over the 3 weeks ranged from 150 to $360 \mathrm{~min}$ (mean $=328 \mathrm{~min}$, see Table 2 for observation time of individual mothers).

\section{Scoring}

Most coded variables were chosen based on their inclusion in past studies examining mothering in dogs (Guardini et al., 2015; Foyer et al., 2016). Moreover, because the rodent literature has successfully tracked different nursing postures and discovered associations with later pup behavior (e.g., Myers et al., 1989b; Liu et al., 2000; Champagne et al., 2003) and because our own observations revealed behavioral differences in nursing postures among the mothers, we diverged from past dog studies that coded nursing as a single category and instead coded distinct types of nursing. The following seven variables were coded from video:

Time in pool: Mother's whole body in whelping pool.

\footnotetext{
${ }^{1}$ For additional information, see this website: http://bit.ly/2h9N3na and these informational videos: https://www.youtube.com/watch?v=7tJeqIJbTjg and https://www.youtube.com/watch?v=iI9MW6Hw5Fk.
} 
TABLE 1 | Mother and litter demographics.

\begin{tabular}{|c|c|c|c|c|c|}
\hline Mother & Breed & Age (months) & Parity & Litter size & Litter breed \\
\hline Della & Labrador Retriever & 49 & 4 & 6 & Labrador-Golden Cross \\
\hline Lizzie & Golden Retriever & 22 & 1 & 9 & Golden \\
\hline Dori & Golden Retriever & 29 & 2 & 5 & Labrador-Golden Cross \\
\hline Lolly & German Shepherd & 37 & 3 & 2 & German Shepherd \\
\hline Maude & Labrador Retriever & 23 & 1 & 9 & Labrador Retriever \\
\hline Ayesha & Labrador Retriever* & 32 & 2 & 10 & Labrador Retriever \\
\hline Foxy & German Shepherd & 31 & 2 & 7 & German Shepherd \\
\hline Toffee & Labrador Retriever & 57 & 5 & 6 & Labrador Retriever \\
\hline Carey & Labrador Retriever & 31 & 2 & 8 & Labrador Retriever \\
\hline Lea & German Shepherd & 49 & 4 & 6 & German Shepherd \\
\hline Leah & German Shepherd & 37 & 3 & 5 & German Shepherd \\
\hline Paris & German Shepherd & 26 & 2 & 4 & German Shepherd \\
\hline Elise & German Shepherd & 23 & 1 & 9 & German Shepherd \\
\hline Xyris & Labrador Retriever & 40 & 3 & 7 & Labrador Retriever \\
\hline Lisa & German Shepherd & 38 & 3 & 4 & German Shepherd \\
\hline Mean and S.D. & & $35.9 \pm 9.2$ & $2.6 \pm 1.0$ & $6.6 \pm 2.2$ & \\
\hline
\end{tabular}

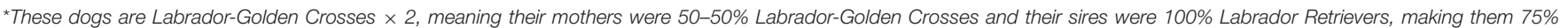
Labrador Retriever. Thus, in all analyses, these dogs were classified as Labrador Retrievers.

TABLE 2 | Mother and litter participation.

\begin{tabular}{|c|c|c|c|c|c|}
\hline Mother & $\begin{array}{l}\text { Observation } \\
\text { time: Week } 1 \\
\text { (minutes) }\end{array}$ & $\begin{array}{l}\text { Observation } \\
\text { time: Week } 2 \\
\text { (minutes) }\end{array}$ & $\begin{array}{c}\text { Observation } \\
\text { time: Week } 3 \\
\text { (minutes) }\end{array}$ & $\begin{array}{c}\text { Reaction to } \\
\text { human testing } \\
\text { (week observed) }\end{array}$ & $\begin{array}{l}\text { Maternal cortisol } \\
\text { (week collected) }\end{array}$ \\
\hline Della & 110 & 0 & 40 & 3 & NA \\
\hline Lizzie & 110 & 0 & 120 & 3 & NA \\
\hline Dagmar & 0 & 120 & 90 & 2,3 & 2 \\
\hline Dori & 120 & 100 & 120 & $1,2,3$ & 1,2 \\
\hline Lolly & 100 & 120 & 120 & $1,2,3$ & 1,2 \\
\hline Dotty & 100 & 120 & 120 & $1,2,3$ & 2 \\
\hline Onyx & 120 & 120 & 80 & $1,2,3$ & 1,2 \\
\hline Maude & 120 & 120 & 120 & $1,2,3$ & 1,2 \\
\hline Ayesha & 120 & 120 & 120 & $1,2,3$ & 1,2 \\
\hline Foxy & 120 & 120 & 120 & $1,2,3$ & 1,2 \\
\hline Toffee & 120 & 120 & 80 & $1,2,3$ & 1,2 \\
\hline Carey & 120 & 120 & 120 & $1,2,3$ & 1,2 \\
\hline Aura & 120 & 120 & 120 & $1,2,3$ & 1,2 \\
\hline Naomi & 120 & 120 & 80 & $1,2,3$ & 1,2 \\
\hline Omega & 120 & 120 & 120 & $1,2,3$ & 1,2 \\
\hline Lea & 120 & 120 & 120 & $1,2,3$ & 1,2 \\
\hline Leah & 120 & 120 & 120 & $1,2,3$ & 1,2 \\
\hline Paris & 120 & 120 & 120 & $1,2,3$ & 1,2 \\
\hline Elise & 120 & 120 & 120 & 2,3 & 1,2 \\
\hline Xyris & 120 & 120 & 120 & 1,2 & 1 \\
\hline Lisa & 120 & 120 & 120 & $1,2,3$ & 1,2 \\
\hline
\end{tabular}


Vertical nursing per pup: Mother nursing (at least one puppy suckling) while standing or sitting in the whelping pool, divided by the number of puppies in her litter.

Lateral nursing per pup: Mother nursing (at least one puppy suckling) while lying on her side or back, so that part or all of her nipples were exposed, divided by the number of puppies in her litter.

Ventral nursing per pup: Mother nursing (at least one puppy suckling) while lying on her stomach, so that her nipples were not easily exposed to the puppies, divided by the number of puppies in her litter.

Contact per pup: Mother lying in close proximity to one or more of her puppies so that the puppies' bodies and/or faces were touching the mother, divided by the number of puppies in her litter.

Licking/grooming per pup: Mother licking, grooming, and/or sniffing her puppies, divided by the number of puppies in her litter.

Orienting out: Mother orienting/looking outside of her pen to the main area of the pavilion. This behavior was only coded when the mother was in the whelping pool with her puppies.

For each of these seven variables, we calculated the average duration in seconds of the behavior during each week's observation sessions. Thus, each of the 21 mothers ended up with one score on all seven variables from each of the 3 weeks (Table 2). Exceptions were Della (missing week 2), Lizzie (missing week 2), and Dagmar (missing week 1).

Coding from video ( $n=68910$-min sessions) was completed by four different observers, with the majority (55\%) coded by EB. Three other observers, each of whom had participated in all aspects of the study, coded $15 \%$ of the videos. Inter-rater reliability was determined by randomly selecting 15 10-min sessions for all observers to code. A high degree of reliability was found for all variables [ICC $(2,4)=0.96-1.00$ with a $95 \%$ confidence interval from 0.89 to 1.00$]$. We were unable to distinguish individual puppies from video, so our observation unit was the litter (as in Foyer et al., 2016).

\section{Statistical Analysis}

All statistical analyses were carried out in $\mathrm{R}$ version 3.3.0 ( $\mathrm{R}$ Development Core Team, 2016). We first examined the raw scores on the seven behavioral variables and tested for rank-order stability over time using Kendall's rank correlation coefficients.

To summarize variation in maternal behavior, we applied principal components analysis (PCA) with orthogonal (varimax) rotation to the seven behavioral variables. We first verified that there was an adequately compact pattern of correlations, indicated by Kaiser-Meyer-Olkin (KMO) measures of sampling adequacy $>0.5$, and that our variables were sufficiently correlated, indicated by a significant Bartlett's test $(p<0.05)$. We determined the number of factors to retain by using parallel analysis (Horn, 1965), fit using the $\mathrm{R}$ package "paran" (Dinno, 2012), as well as the Comparison Data technique (Ruscio and Roche, 2012). Each mother received a score on each principal component on weeks 1,2 , and 3 . We tested for rank-order stability in principal component scores over time using Pearson correlation coefficients.

Finally, because breed, litter size, birth season, parity, and litter sex ratio have been identified in previous studies as potentially affecting mother and puppy behavior, we included each of these parameters as covariates in the regression model described below. Mother's age was not included as it was redundant with parity $(r=0.97, p<0.001)$. To test for associations between these covariates and the single factor, Maternal behavior, that best explained our data, we used a Generalized Estimating Equations version of a general linear regression model (GEE-GLM) to estimate adjusted associations of breed, litter size, birth season, parity, and litter sex ratio with Maternal behavior. We used a Gaussian error distribution with litter as the unit of analysis. Variance estimates for the statistical tests on the regression coefficients were adjusted for clustering due to litter effects using generalized estimating equations (Liang and Zeger, 1993). All models were fit using the R package "geepack" (Halekoh et al., 2006). To compare models, we used the R package "anova" in the "stats" package.

\section{Results}

\section{Stability of Maternal Behavior over Time}

As in previous studies (e.g., Foyer et al., 2016), the average of all seven variables of maternal care decreased over time. With few exceptions, the rank orders of mothers on the different behaviors were significantly correlated (i.e., stable) from weeks 1 to 2 and 2 to 3 (Table 3 ).

\section{Principal Components Analysis}

A KMO test was conducted on the seven behavioral variables listed above. The sampling adequacy for the analysis was $\mathrm{KMO}=$ 0.69 . All KMO values for individual variables were $>0.54$, which is above the acceptable limit of 0.5 (Field et al., 2012). Bartlett's test, $\chi^{2}(21)=221, p<0.001$, indicated that correlations between items were sufficiently large for PCA. Furthermore, all items were correlated to at least the level of 0.49 with at least one other item, suggesting reasonable factorability. Parallel analysis using 5000 iterations recommended retaining one principal component, as did the Comparison Data technique. The scree plot showed inflexions that were consistent with retaining a one-component solution. The total variance explained by this factor was $54 \%$ (Table 4).

Loadings of the behavioral variables onto the principal component (Maternal behavior) suggested that mothers who scored high on this component were often present and interactive with their puppies. They spent a considerable amount of time in the pool, were in frequent contact with their puppies, and showed high levels of oral behavior toward their puppies, including anogenital licking, grooming of the face and body, and sniffing. They often nursed them from a lateral position that provided puppies with the easiest access to their milk, but also engaged in nursing from a ventral or vertical position that made it more labor intensive for puppies to nurse effectively. They also were often attentive toward the main pavilion rather than toward their puppies (orienting out), despite being in the pool with them. 
TABLE 3 | Consistency of maternal behavior across weeks.

\begin{tabular}{|c|c|c|c|}
\hline Behavior & $n$ & Time frame & Kendall's tau \\
\hline \multirow[t]{2}{*}{ Time in pool } & 18 & Weeks 1-2 & $0.341^{*}$ \\
\hline & 19 & Weeks 2-3 & $0.590^{\star \star \star}$ \\
\hline \multirow[t]{2}{*}{ Contact per pup } & 18 & Weeks 1-2 & 0.245 \\
\hline & 19 & Weeks 2-3 & $0.419^{*}$ \\
\hline \multirow[t]{2}{*}{ Licking/grooming per pup } & 18 & Weeks 1-2 & 0.305 \\
\hline & 19 & Weeks 2-3 & $0.371^{*}$ \\
\hline \multirow[t]{2}{*}{ Lateral nursing per pup } & 18 & Weeks 1-2 & 0.322 \\
\hline & 19 & Weeks 2-3 & $0.430^{*}$ \\
\hline \multirow[t]{2}{*}{ Ventral nursing per pup } & 18 & Weeks 1-2 & 0.302 \\
\hline & 19 & Weeks 2-3 & 0.033 \\
\hline \multirow[t]{2}{*}{ Vertical nursing per pup } & 18 & Weeks 1-2 & $0.462^{*}$ \\
\hline & 19 & Weeks 2-3 & $0.624^{\star \star}$ \\
\hline \multirow[t]{2}{*}{ Orienting out } & 18 & Weeks 1-2 & $0.541^{\star \star}$ \\
\hline & 19 & Weeks 2-3 & 0.274 \\
\hline
\end{tabular}

${ }^{\star \star *} p<0.001,{ }^{* *} p<0.01,{ }^{*} p<0.05, \cdot p<0.10$.

\section{Stability of Maternal Behavior over Time}

The rank orders of scores on Maternal behavior were significantly correlated (i.e., stable) from weeks 1 to $2(r=0.82, p<0.001)$ and 2 to $3(r=0.89, p<0.001)$.

\section{Potential Covariates}

We conducted a GEE-GLM with breed (a categorical variable), litter size (2-10), birth season (a binary variable consisting of spring versus winter), parity (1-5), and litter sex ratio (percent male) as the predictors and Maternal behavior as the dependent measure (Table 5). Maternal behavior was not related to birth season or litter sex ratio. However, Labrador Retriever mothers demonstrated significantly higher Maternal behavior scores than German Shepherds $(\beta=0.56, S E=0.19, p=0.003)$. Additionally, mothers with smaller litters had significantly higher Maternal behavior scores than mothers with larger litters $(\beta=-0.32, S E$ $=0.07, p<0.001$ ), and mothers who had whelped fewer litters had significantly higher Maternal behavior scores than more experienced mothers $(\beta=-0.16, S E=0.07, p=0.02)$.

"Contact per pup" was the behavior that loaded most strongly onto Maternal behavior, and it was also associated with (a) litter size: mothers with smaller litters had more contact per pup than mothers with larger litters $(\beta=-0.31, S E=0.06, p<0.001)$, (b) breed: Labrador Retriever mothers had more contact per pup with their litters than German Shepherd mothers $(\beta=0.56, S E=$ $0.15, p<0.001)$, and (c) parity: mothers who had whelped fewer litters had more contact with their pups than more experienced mothers $(\beta=-0.22, S E=0.07, p<0.001)$. The loading of "contact per pup" was not just an artifact of these covariates, however, because a model that used Maternal behavior score along with litter size, parity, and breed to predict the frequency with which a mother lay in contact per pup was significantly
TABLE 4 | Components and loadings of the PCA over the observations of maternal care.

\begin{tabular}{lc}
\hline Observation variable & Maternal behavior \\
\hline Time in pool & 0.72 \\
Contact per pup & 0.90 \\
Licking/grooming per pup & 0.72 \\
Lateral nursing per pup & 0.84 \\
Vertical nursing per pup & 0.54 \\
Ventral nursing per pup & 0.63 \\
Orienting out & 0.74 \\
Eigenvalue & 3.78 \\
Proportion of variance explained & 0.54
\end{tabular}

better than a model that used only litter size, parity, and breed [ANOVA model comparison, $\chi^{2}(1)=30.2, p<0.001$ ]. In other words, mothers who scored high on Maternal behavior were in contact with their puppies more frequently than would have been expected based only on their litter size, breed, and parity.

We therefore concluded that the principal component was not redundant with breed, litter size, birth season, parity, or sex ratio. However, because some associations were found with these covariates, we included them as potential confounding variables in subsequent models.

In sum, we tracked seven maternal care behaviors of 21 mothers toward their litters over the first 3 weeks postwhelping. The mothers' interactions were best explained by one principal component of maternal style, which was stable over time. Maternal behavior was not explained by birth season or sex ratio of the litter. While Maternal behavior was associated with breed, litter size, and parity, this component still provided additional information above and beyond that provided by those demographic variables alone.

\section{PART 2: EXPERIMENTAL TEST OF MATERNAL STYLE}

\section{Methods}

Assuming that Maternal behavior captured individual variation in maternal style that was consistent over time, we hypothesized that it should also be associated with other measures of maternal behavior that were collected concurrently but were not used to generate the principal component. To that end, we conducted a 5 min preference test that sought to quantify a mother's attentiveness to her puppies in the face of a competing social interest: a familiar human in the whelping pen. The design of this experiment was meant to evoke the mothers' typical interactions with kennel staff, since staff members entered each pen multiple times per day. The goal was, first, to place the mothers in a situation where they would have to choose between their puppies and an inviting distraction, and then to determine if the mothers' choices could be predicted based on their mothering style as measured by their scores on the PC. 
TABLE 5 | Results of a GEE-GLM in which the dependent variable was Maternal behavior.

\begin{tabular}{|c|c|c|c|}
\hline Predictor variables & Estimate & SE & $p$-value \\
\hline Intercept & 2.43 & 0.52 & $<0.001^{\star \star \star}$ \\
\hline Golden Score & -0.11 & 0.31 & 0.716 \\
\hline Labrador Score & 0.56 & 0.19 & $0.003^{\star \star}$ \\
\hline Birth season & 0.35 & 0.28 & 0.215 \\
\hline Litter sex ratio & -0.53 & 0.40 & 0.184 \\
\hline Litter size & -0.32 & 0.07 & $<0.001^{\star \star \star}$ \\
\hline Parity & -0.16 & 0.07 & $0.018^{\star}$ \\
\hline
\end{tabular}

Predictor variables were Golden score, Golden Retriever compared to German Shepherd; Labrador score, Labrador Retriever compared to German Shepherd; birth season, spring or winter; litter sex ratio, percent male; litter size, 2-10; and parity, 1-5). Mother ID was entered as a random effect. $N=17$ mothers (week 1) and 18 mothers (week 2). Statistical tests of significance use GEE. ${ }^{* \star *} p<0.001,{ }^{* *} p<0.01,{ }^{*} p<0.05$.

\section{Subjects and Procedure}

The same mothers that were observed above participated in this experiment, conducted once a week during their first $(n=17$ litters), second ( $n=19$ litters), and third ( $n=21$ litters) weeks postpartum (see Table 2).

All experiments took place between 8:45 and 18:30. When the mother was in the pool nursing at least one puppy and no other humans were in the pavilion, the experimenter set up the camera outside of her pen, started the camera, and left the area. One minute later, the experimenter returned. First, she greeted the dog verbally by name once, outside of the gate. Immediately afterwards, she opened the gate and greeted the dog again from inside the pen. Then for $1 \mathrm{~min}$ she stood in the pen, facing toward the pool from $\sim 2$ feet away, in a relaxed posture. If the mother left the pool and interacted with the experimenter during this time, the experimenter responded by petting and talking to the mother. For the next minute, the experimenter sat on the ground in the same spot. Again, the experimenter interacted with the mother if the mother approached her. Finally, for the last minute, the experimenter remained sitting where she was but hid her face and actively ignored the mother. After a total of $3 \mathrm{~min}$ had elapsed, the experimenter exited the pen and the session ended. The experimenter was always one of three females of similar age, with the first author (EB) playing the role of experimenter in $95 \%$ of all sessions.

\section{Scoring}

The following three variables were coded from video:

Initial approach: Time elapsed (in seconds) between the experimenter's first greeting and when all four of the mother's paws were outside of the whelping pool. In order to approach the human, the mother had to stop nursing and leave her puppies. A low time corresponded with the mother being least interested in her puppies while in the presence of the familiar human, while a high time corresponded to the mother being most invested in remaining with her puppies. If a mother never left her puppies over the course of the experiment, she received the maximum score of $180 \mathrm{~s}$.
Contact with human: Duration (in seconds) that the mother was outside of the pool and sniffing or touching the experimenter with any part of her body, ranging from 0 to $168 \mathrm{~s}$.

Time with puppies: Percent of the 3 min session that the mother remained in the pool with her puppies (all four legs in pool) while the experimenter was in the pen, ranging from 0 to $100 \%$.

Each of the 21 mothers received scores on all three variables from each of the 3 weeks, with the following exceptions: Della (missing weeks 1 and 2), Lizzie (missing weeks 1 and 2), Dagmar (missing week 1), Elise (missing week 1), and Xyris (missing week 3; Table 2).

All coding was completed by an observer who had not participated in data collection. To assess reliability of the videocoded measures, EB then coded $20 \%$ of randomly selected trials. Ratings on initial approach and time with puppies were correlated at $r_{\tau}=1(p<0.001)$, and ratings on contact with human were correlated at $r_{\tau}=0.94(p<0.001)$.

\section{Statistical Analysis}

All statistical analyses were carried out in in $\mathrm{R}$ (version 3.3.0, R Foundation for Statistical Computing, R Development Core Team, 2016). The three outcome variables were standardized using a $z$-score because they used different scales. Mothers received a score on each outcome variable during weeks $1(n=$ $17), 2(n=19)$, and $3(n=21)$. We used Pearson correlation coefficients to determine how associated the outcome measures were, and found them all highly correlated $(r>0.90)$. We therefore chose one and tested for rank-order stability on that measure over time using a Pearson correlation coefficient. We then used a GEE-GLM that included the data for each week and used litter as the unit of observation to test whether Maternal behavior was associated with a mother's relative interest in her puppies, as measured by our one outcome variable. We included as predictors an interaction, Maternal behavior by week, as well as breed, litter size, birth season, parity, and litter sex ratio. We then used a backward-selection strategy and retained all confounders that influenced the association of interest by at least $10 \%$.

\section{Results}

\section{Outcome Variables}

All three outcome variables were highly correlated with one another. "Initial approach" was correlated with "Contact with human": $r=-0.91, p<0.001$ and with "Time with puppies": $r=0.95, p<0.001$. "Contact with human" was correlated with "Time with puppies": $r=-0.94, p<0.001$. In other words, mothers who were slow to initially approach the experimenter also spent short amounts of time in contact with the experimenter and a considerable amount of the 3 min experimental session in the pool with their puppies. Because "Time with puppies" was the variable most highly correlated with the other two, we used that measure as the dependent variable in subsequent analyses.

The rank order scores of mothers on "Time with puppies" from weeks 1 to 2 were positively associated $(r=0.28)$, but not 
significant $(p=0.30)$. The same pattern held true from weeks 2 to $3(r=0.16, p=0.50)$.

\section{Maternal Interest in Puppies during the Experiment}

We were interested in evaluating if our PC, Maternal behavior, was associated with this experimental measure of a mother's relative interest in her puppies, as measured by her "Time with puppies" score.

We used a GEE-GLM with "Time with puppies" as the dependent variable, Maternal behavior as a predictor variable, and mother ID as a random effect. We also included an interaction, Maternal behavior by week, to test whether the relationship between maternal style and a mother's reaction to a human visitor changed as the puppies got older. Finally, we included breed, litter size, birth season, parity, and litter sex ratio as covariates. We were able to remove litter sex ratio and litter size from the final model, as they were determined to be non-significant and non-confounding (Table 6).

The interaction was significant $(\beta=0.58, S E=0.22, p<$ 0.01 ). Specifically, during week 2, mothers' scores on Maternal Behavior predicted how much time they spent with their puppies vs. the human (high scores predicted high preference for puppies; Week $2 \beta=0.43, S E=0.22, p=0.05)$. There was no significant association during weeks 1 or 3 (Week $1 \beta=0.05, S E=0.13, p=$ 0.71 ; Week $3 \beta=-0.15, S E=0.16, p=0.33)$.

In sum, we conducted a weekly experiment to measure mothers' attention to their puppies in the face of a competing social interest. Mothers' responses were best characterized by her "Time with puppies" score. Mothers who scored high on Maternal behavior, i.e., those that regularly spent the most time in the whelping pool physically contacting, nursing, and grooming their puppies in their daily lives, also spent the most time with their puppies during week 2 of the experiment.

\section{PART 3: SALIVARY CORTISOL REACTION TO A STRESSOR}

\section{Methods}

To test the hypothesis that our PC, Maternal behavior, was associated with a measure of maternal stress, we compared this variable to salivary cortisol levels taken from the mother during the first 2 weeks after birth. Cortisol was collected before each mother experienced a brief 5 min separation from half of her litter, as well as 20 and $40 \mathrm{~min}$ after the separation. This scenario mimicked a potentially stressful, albeit typical event, as staff members removed neonatal puppies individually or in small groups to weigh them each day. This separation and the accompanying saliva collections were conducted on 2 nights each week.

We opted to measure cortisol from saliva because it is a noninvasive alternative to drawing blood (Beerda et al., 1996). Saliva collection closely adhered to previously published methods (Dreschel and Granger, 2009). While one or two experimenters held the dog in place, the main experimenter (EB) wore latex gloves and held a Salimetrics ${ }^{\circledR}$ Children's Swab under the dog's tongue and around the dog's cheek pouches, avoiding the gums, for 2-6 min. The swab was gently moved and repositioned
TABLE 6 | Results of a GEE-GLM in which the dependent variable was "Time with puppies."

\begin{tabular}{lrcc}
\hline Predictor variables & Estimate & SE & p-value \\
\hline Intercept & 1.89 & 0.34 & $<0.001^{\star \star \star}$ \\
Week 2 & -0.94 & 0.24 & $<0.001^{\star \star \star}$ \\
Week 3 & -1.16 & 0.29 & $<0.001^{\star \star \star}$ \\
Golden Score & 0.02 & 0.31 & 0.937 \\
Labrador Score & -0.33 & 0.22 & 0.136 \\
Parity & -0.34 & 0.11 & $0.002^{\star \star}$ \\
Birth season & -0.58 & 0.27 & $0.013^{\star}$ \\
Interaction & 0.58 & 0.22 & $0.007^{\star *}$ \\
Maternal behavior $\times$ Week 1 & 0.05 & 0.13 & 0.710 \\
Maternal behavior $\times$ Week 2 & 0.43 & 0.22 & $0.053^{\star}$ \\
Maternal behavior $\times$ Week 3 & -0.15 & 0.16 & 0.334 \\
\hline Predictor & & &
\end{tabular}

Predictor variables were Maternal behavior; week (1, 2, and 3); Golden score, Golden Retriever compared to German Shepherd; Labrador score, Labrador Retriever compared to German Shepherd; parity, 1-5; and birth season, spring or winter. Mother ID was entered as a random effect. $N=17$ mothers (week 1), 19 mothers (week 2), and 21 mothers (week 3). Statistical tests of significance use GEE. ${ }^{* *} p<0.001,{ }^{* *} p<0.01$, ${ }^{*} p<0.05$.

throughout sampling, and then placed into a labeled storage tube. Past research indicates that handling of a dog during saliva collections lasting up to $5 \mathrm{~min}$ does not influence the cortisol concentration (Kobelt et al., 2003; Coppola et al., 2006), and in our own data mean cortisol levels were not correlated with duration of collection $(r=0.002, p=0.97)$.

Post-collection, all samples were either refrigerated (at $4^{\circ} \mathrm{C}$ ) and then centrifuged, or placed directly into the Triac ${ }^{\mathrm{TM}}$ centrifuge for $15 \mathrm{~min}$ at $1500 \mathrm{x} g$ rotation. After centrifugation and no more than $95 \mathrm{~min}$ after collection, samples were stored in a freezer (at $-20^{\circ} \mathrm{C}$ ) until 1-6 months later, when they were sent for analysis to Arizona State University's Institute for Interdisciplinary Salivary Bioscience Research.

\section{Subjects and Procedure}

We obtained samples from mothers during their first $(n=17$ mothers) and second ( $n=18$ mothers) weeks post-whelping (Table 2). Thus, all mothers who participated in the maternal separation experiment gave saliva on 4 separate days, with the exception of Dagmar (missing week 1), Lolly (missing day 2), Dotty (missing week 1), Onyx (missing days 2 and 4), Maude (missing day 2), Elise (missing day 4), and Xyris (missing day 2 and week 2).

Ten minutes prior to saliva collection, water was temporarily removed from the pen to avoid dilution of saliva. EB first collected baseline saliva between 20:00 and 21:00, $\sim 2-3 \mathrm{~h}$ after the mother's evening meal, when she was in the pen with all of her litter. Between 21:00 and 23:00, an experimenter returned and removed half of her litter by placing them in a towel-lined tub and carrying them out of sight to the examination room located within the pavilion. Five minutes later, the experimenter returned the puppies to the mother. Then, 20 and $40 \mathrm{~min}$ after the initial removal, EB returned to collect post-stressor saliva from the mother. After the third saliva collection, water was returned to the mother and she received a Milk-Bone ${ }^{\circledR}$ biscuit. 
Samples $(N=212$, drawn from 19 dogs $)$ were mailed on dry ice, then thawed and assayed for salivary cortisol using an enzyme immunoassay kit at the Institute for Interdisciplinary Salivary Bioscience Research. With the exception of one sample assayed as a singlet, all samples were assayed in duplicate using $50 \mu \mathrm{L}$ of saliva, and the average of these two measures were used in subsequent analyses. The lower limit of detection was $0.007 \mu \mathrm{g} / \mathrm{dl}$, and the average coefficient of variation for the assay was $3.0 \%$. Analysis was repeated for five samples that had a coefficient of variation $>15 \%$.

\section{Scoring}

Prior to analysis, we applied a natural log transformation to all cortisol measurements. We also created a peak cortisol score for each mother on every collection day. Past studies in dogs and humans suggest that there is inter-individual variation in time to reach peak salivary cortisol levels (e.g., Beerda et al., 1996; LopezDuran et al., 2009). Thus, the peak cortisol score was calculated by subtracting each mother's baseline pre-stressor cortisol measure from her highest post-stressor cortisol measure, taken either 20 or $40 \mathrm{~min}$ after the removal of her puppies.

\section{Statistical Analysis}

All statistical analyses were carried out in $\mathrm{R}$ (version 3.3.0, $\mathrm{R}$ Foundation for Statistical Computing, R Development Core Team, 2016). We first determined the best way to group the cortisol results by using a linear mixed model (LMM) to examine trends over time. We then tested if the mothers' baseline stress levels were associated with Maternal behavior by using a GEE-GLM. Finally, we tested if removing the puppies elicited a significant stress response across mothers by conducting a paired-samples $t$-test to compare the mean of the baseline scores against the mean of the peak scores. Upon finding a significant difference, we used a GEE-GLM to test whether the mothers' stress responses were associated with Maternal behavior. As before, we adjusted for confounding variables by using a backward-selection strategy and retained all confounders that influenced the association of interest by at least $10 \%$.

\section{Results}

\section{Stability of Cortisol over Time}

To determine if a mother's baseline cortisol levels differed by collection day, we used a LMM with baseline cortisol as the dependent variable, day (1 through 4 ) as the predictor variable, and $\operatorname{dog}$ ID as a random effect. Once again, day was not significant, indicating that mothers' baseline scores were similar across days.

We therefore created a Week 1 baseline cortisol score by taking the average scores across days 1 and 2 and a Week 2 baseline cortisol score by taking the average scores across days 3 and 4 . For the four cases in week 1 and two cases in week 2 where a mother had only a single baseline score, we used that score alone.

To determine if a mother's peak cortisol response differed by collection day, we used peak cortisol score as the dependent variable, day (1 through 4 ) as the predictor variable, and dog ID as a random effect. Day was again not significant, indicating that mothers' cortisol responses were similar across all days.

This similarity in peak cortisol scores within weeks justified the creation of Week 1 and Week 2 average peak cortisol scores. For the three cases in week 1 and one case in week 2 where a mother had only a single peak cortisol score, we used that score alone.

\section{Baseline Maternal Stress Levels}

We first asked if a mother's baseline cortisol scores were associated with Maternal behavior. We used a GEE-GLM with baseline cortisol score as the dependent variable, Maternal behavior as a predictor variable, and mother ID as a random effect. We also included an interaction of Maternal behavior by week, and breed, litter size, birth season, parity, and litter sex ratio as covariates. The interaction was nonsignificant, so it was dropped from the model. We were also able to exclude week, parity and litter sex ratio from the final model (Table 7).

We found a marginally significant main effect $(\beta=0.16, S E$ $=0.09, p=0.06$ ): mothers who spent more time engaging in maternal behaviors also had higher levels of baseline cortisol over weeks 1 and 2.

\section{Maternal Response to Separation}

To verify that removing half of the puppies was indeed a stressful event for mothers, we compared the means of mothers' baseline cortisol scores to the means of mothers' peak cortisol scores. There was a significant difference between baseline scores $(M=$ $-1.73, S D=0.41)$ and peak cortisol scores $(M=0.28, S D=0.39)$; $t_{(34)}=21.28, p<0.001$.

Next, we investigated if a mother's anxiety when temporarily separated from half of her litter, as measured by her peak cortisol score collected during this event, was associated with the maternal style PC.

We used a GEE-GLM with the peak cortisol score as the dependent variable, Maternal behavior as a predictor variable, and mother ID as a random effect. We also included an interaction, Maternal behavior by week, to explore whether the relationship between a mother's investment in her puppies and her endocrine response differed from week 1 to 2 . We also included breed, litter size, birth season, parity, and litter sex ratio as covariates, but were able to exclude parity and litter size from our final model (Table 8). We found a significant interaction ( $\beta$ $=-0.23, S E=0.09, p=0.013)$ : mothers who spent more time engaging in maternal behaviors tended toward higher levels of peak cortisol over week $1(\beta=0.20, S E=0.11, p=0.06)$, but this association disappeared by week two $(\beta=-0.03, S E=0.07, p=$ $0.68)$.

To test the hypothesis that differences in maternal peak cortisol response were not simply a function of differences in maternal baseline cortisol levels, we ran the same full model as above but also included baseline cortisol as a predictor. Baseline was not significant in this model, and all other findings were consistent with our previous model, indicating that peak cortisol response was not just an artifact of baseline cortisol levels.

In sum, we measured maternal stress levels before and after a slightly stressful event. Pre-stressor, baseline salivary cortisol 
TABLE 7 | Results of a GEE-GLM in which the dependent variable was the baseline cortisol score.

\begin{tabular}{lccc}
\hline Predictor variables & Estimate & SE & $\boldsymbol{p}$-value \\
\hline Intercept & -2.21 & 0.28 & $<0.001^{\star \star \star}$ \\
Maternal behavior & 0.16 & 0.09 & 0.061 \\
Golden Score & 0.20 & 0.30 & 0.498 \\
Labrador Score & 0.07 & 0.17 & 0.664 \\
Litter Size & 0.05 & 0.05 & 0.269 \\
Birth season & 0.16 & 0.17 & 0.329
\end{tabular}

Predictor variables were Licking/grooming; Golden score, Golden Retriever compared to German Shepherd; Labrador score, Labrador Retriever compared to German Shepherd; litter size, 2-10; and birth season, spring or winter. Mother ID was entered as a random effect. $N=17$ mothers (week 1) and 18 mothers (week 2). Statistical tests of significance use GEE. ${ }^{* \star *} p<0.001, p<0.10$.

TABLE 8 | Results of a GEE-GLM in which the dependent variable was the peak cortisol score.

\begin{tabular}{lrrr}
\hline Predictor variables & Estimate & SE & $\boldsymbol{p}$-value \\
\hline Intercept & 0.00 & 0.17 & 0.991 \\
Week & -0.02 & 0.14 & 0.900 \\
Golden Score & 0.08 & 0.15 & 0.605 \\
Labrador Score & -0.14 & 0.10 & 0.146 \\
Birth season & 0.19 & 0.10 & $0.046^{*}$ \\
Litter sex ratio & 0.49 & 0.24 & $0.041^{*}$ \\
Interaction & & & $0.013^{*}$ \\
Maternal behavior $\times$ Week 1 & 0.20 & 0.11 & 0.063 \\
Maternal behavior $\times$ Week 2 & -0.03 & 0.07 & 0.675 \\
& & &
\end{tabular}

Predictor variables were Licking/grooming, week (1 or 2); Golden score, Golden Retriever compared to German Shepherd; Labrador score, Labrador Retriever compared to German Shepherd; litter sex ratio, percent male; and birth season, spring or winter. Mother ID was entered as a random effect. $N=17$ mothers (week 1) and 18 mothers (week 2). Statistical tests of significance use GEE. ${ }^{*} p<0.05, \cdot p<0.10$.

of the mothers over weeks 1 and 2 was positively associated with Maternal behavior. We also found that maternal peak salivary cortisol, or the maximum amount that a mother's cortisol levels increased from baseline levels following temporary puppy removal, was marginally associated with Maternal behavior. Specifically, mothers who ranked high on contact, proximity, nursing, grooming, and orienting out in both weeks showed the largest peaks in salivary cortisol after being briefly separated from half of their litter.

\section{GENERAL DISCUSSION}

Consistent with results from previous studies (Foyer et al., 2016; Guardini et al., 2016), our observational data on seven measures of maternal style were best summarized by one principal component, Maternal behavior. Mothers who scored high on this component were present (often in close proximity and contact with their puppies), interactive (engaged in high levels of licking, grooming, and lateral, ventral, and vertical nursing), and vigilant (displayed high levels of orienting out into the main pavilion). This PC was also associated with concurrent behavioral and stress-related measures of mothering, results that have not been documented previously.

We validated our approach in several ways. First, mothers were consistent in their rank order on the PCs over time despite group-level decreases in maternal care as the weeks progressed. This stability gave us confidence that the PC captured meaningful and enduring individual differences. Results are also encouraging because they mirror data from the human and non-human literature, in which studies have found stable maternal differences over time (e.g., Berman, 1990; van IJzendoorn et al., 2000; Pittet et al., 2014).

Second, scores on PCs were not simply redundant with other measures, such as breed, litter size, birth season, parity, or litter sex ratio. If measures of maternal style had not provided any additional information beyond that provided by the demographic characteristics of mothers and their litters, then the extra time spent observing the mothers and building the profiles would not have been justified.

That being said, we did find some associations between our demographic covariates and Maternal behavior. In line with the results of Foyer et al. (2016), we found a significant effect of litter size, wherein females with smaller litters displayed higher scores on Maternal behavior. This is not surprising, since it is physically difficult for a mother of 10 to interact with each individual puppy at the same rate as a mother of two. Furthermore, tending to larger litters is likely more demanding and exhausting, potentially leading the mother to spend less time with them. Priestnall (1972) proposed that these aversive factors might be the reason that mice mothers of large litters spend less time in the nest than mothers of small litters, after finding that the difference could not be entirely explained by unequal nutritional requirements.

We also found an effect of parity: the more experienced the mother was, the less maternal behavior she displayed. In the only other study to examine the effect of parity on motherpup interaction, Guardini et al. (2015) found that primiparous mothers increased their quality of care over time, surpassing multiparous mothers only in week 3 , while multiparous mothers stayed relatively constant in their amount of care.

We were particularly interested in examining the effect of breed on Maternal behavior, as past studies have limited their sample to a single breed (Foyer et al., 2016; Guardini et al., 2016). We did find a breed difference among levels of Maternal behavior, since Labrador Retriever mothers spent more time engaging in maternal behavior than did German Shepherd mothers. No differences were found between Golden Retrievers and the other breeds, a result that might have been due in part to the small sample of this breed in our study.

In a third analysis that validated our approach, we found that the Maternal behavior PC had predictive validity, both in an experiment designed to test temporary maternal preference for puppies versus a familiar human, and in measures of maternal stress. Specifically, in an experiment designed to capture maternal preferences, we found a significant, time-dependent relationship between Maternal behavior and a mother's willingness or lack thereof to stay with her puppies in the presence of a familiar human, a behavioral measure that was not incorporated into the PC. Mothers who scored high on Maternal behavior in week 2 
showed a high motivation to remain with their puppies, despite the presence of a human in the pen. This association was not present in the first and third weeks. The lack of an association during week 1 might be due to a ceiling effect: only 4 of the 17 mothers left the pool at all during the first week.

One limitation of this experimental measure is that not all dogs are equally social. Thus, if a female is not very socially motivated, she might stay in the pool with her pups even if her "maternal urge" is not very strong. However, in this population, we do not believe this concern affected our results. We found that, as expected, females who exhibited high levels of Maternal behavior were indeed more likely to stay with their puppies rather than visiting the human. One possible reason is that all of the dogs at The Seeing Eye have been bred over multiple generations for a friendly, human-oriented demeanor, whereas much less intense directional selection has been applied to mothering style.

In a fourth and final experimental validation of the predictive value of our components, we found two relationships between the $\mathrm{PC}$ and an independent physiological measure. First, we found a marginally significant positive association between Maternal behavior and higher baseline cortisol levels over weeks 1 and 2. This result suggests a link between high levels of maternal care and anxiety, although it is not yet known if this association might be detrimental to future litter outcomes. Additionally, we found an association between Maternal behavior and a measure of maternal stress response measure. Mothers who scored high on Maternal behavior in week 1 showed a larger stress response after half of their litter was temporarily removed, suggesting that puppy removal was particularly stressful for them. These findings are particularly interesting as past research has mainly focused on how maternal style influences later stress responses of the pups themselves (e.g., Francis et al., 1999; Myers et al., 1989a), and has investigated to a lesser extent how the stress of the mother might influence her maternal style (e.g., Champagne and Meaney, 2006). Moreover, these topics have primarily been explored in rodents.

Notably, we found the strongest associations between Maternal behavior and experimental measures of behavior and physiology during the first and second week postpartum. This result is consistent with findings from the rodent literature, where the first week after birth seems to have a particularly important effect on subsequent offspring behavior. In rats, for example, week 1 is the only week during which dams differ in their amount of licking and grooming (Champagne et al., 2003).

Although this study suggests that mothering style in dogs is consistent across weeks, we do not yet know if mothering style is consistent across litters. Data from rodents (Champagne et al., 2003), primates (Fairbanks, 1996), and sheep (Dwyer and Lawrence, 2000) suggest that maternal style is consistent across parturitions. It also remains to be determined how much the surrounding environment affects mothering profiles, as well as if and how maternal style can be manipulated to promote better offspring behavior, temperament, and cognition. In birds (Pittet et al., 2014) and macaques (Maestripieri, 1993), maternal style appears to be correlated with behavioral measures of the mother thought to be associated with temperament. We do not yet know if temperament in dogs can similarly be used to estimate maternal investment.

One surprising finding was the fact that all three nursing styles loaded onto the same component, although lateral nursing loaded the highest (Table 4). We had initially coded them separately because they were a striking feature of our preliminary observations, and because studies of rodents have distinguished three types of nursing (e.g., Myers et al., 1989b; Champagne et al., 2003). Importantly, only arched-back nursing has been consistently associated with later positive outcomes. Rat pups raised by high licking-grooming (LG) and arched-back nursing $(\mathrm{ABN})$ mothers exhibited decreased startle responses and more exploratory behaviors in novel environments (e.g., Liu et al., 1997; Caldji et al., 1998). They also differed in measures of cognition; high LG-ABN pups exhibited superior object recognition (Meaney, 2001; Fish et al., 2004) and were faster and more efficient at problem-solving in a task for spatial memory (Liu et al., 2000) when compared with low LG-ABN pups. One hypothesis argues that these positive outcomes derive from the high levels of tactile simulation during the frequent nipple switching that occurs specifically during arched-back nursing (Liu et al., 2000). Arched-back nursing is also highly correlated with licking-grooming in rodents (Meaney, 2001), making it difficult to disentangle the separate effects; in fact, arched-back nursing is rarely measured by itself. Among the dogs in our study, however, vertical nursing and licking/grooming were only weakly correlated at 0.34 . Dogs, therefore, may provide an opportunity to examine the separate roles of arched-back nursing and lickinggrooming in affecting later behavior, as well as the effects of the three different nursing styles (Bray et al., unpublished data).

Several important questions remain. Now that we have established a reliable characterization of maternal behavior, we plan to test specific hypotheses about its effects on the later behavior of puppies, as well as investigating how enduring those effects may be. Recent studies of maternal care in dogs have found associations between higher levels of early care and increased exploratory tendencies and lower stress responses at 8 weeks (Guardini et al., 2016), as well as increased social engagement, physical engagement, and aggression at 15-18 months (Foyer et al., 2016). Long-term effects of maternal care have also been documented in primates; rhesus monkeys with rejecting mothers were more anxious at 2 years old (Maestripieri et al., 2006), and were also more independent than peers raised by protective mothers (Bardi and Huffman, 2002). Even in our own species, protective (DeVore and Ginsburg, 2005) and harmful (Repetti et al., 2002; Hoeve et al., 2009) effects on later adolescent behavior can be traced, at least in part, back to parenting style. In addition to these behavioral effects, early parental care has been linked to morphological changes in the brain. For example, one study in humans found that having warm, available parents at age four was correlated with hippocampal volume at age 14 (Rao et al., 2010). In addition to puppy temperament outcomes, future analyses will explore maternal effects on later cognition and problem-solving skills, as well as the ultimate success rates of puppies in The Seeing Eye ${ }^{\circledR}$ program. 


\section{AUTHOR CONTRIBUTIONS}

EB, RS, DC, and JS designed the observational and experimental procedures. EB conducted the observations, experiments, and coded the majority of the videos. EB, RS, and MS performed the statistical analyses. EB was the primary author, with assistance from RS and DC and comments from JS and MS.

\section{FUNDING}

This work was supported in part by the University of Pennsylvania Department of Psychology's Norman Anderson Graduate Student Fund, a University of Pennsylvania University Research Foundation award, the Class of 1971 Robert J. Holtz Endowed Fund for Undergraduate Research, and a National Science Foundation Graduate Research Fellowship to EB (DGE-1321851). Any opinions, findings, and conclusions or recommendations expressed in this material are solely the

\section{REFERENCES}

Aisa, B., Tordera, R., Lasheras, B., Del Río, J., and Ramírez, M. J. (2008). Effects of maternal separation on hypothalamic-pituitary-adrenal responses, cognition and vulnerability to stress in adult female rats. Neuroscience 154, 1218-1226. doi: 10.1016/j.neuroscience.2008.05.011

Bardi, M., and Huffman, M. (2002). Effects of maternal style on infant behavior in Japanese macaques (Macaca fuscata). Dev. Psychobiol. 41, 364-372. doi: $10.1002 /$ dev. 10065

Beerda, B., Schilder, M. B., Janssen, N. S., and Mol, J. A. (1996). The use of saliva cortisol, urinary cortisol, and catecholamine measurements for a noninvasive assessment of stress responses in dogs. Horm. Behav. 30, 272-279. doi: 10.1006/hbeh.1996.0033

Berman, C. M. (1990). Consistency in maternal behavior within families of freeranging rhesus monkeys: an extension of the concept of maternal style. Am. J. Primatol. 22, 159-169. doi: 10.1002/ajp.1350220303

Borchelt, P. L. (1983). Aggressive behavior of dogs kept as companion animals: classification and influence of sex, reproductive status and breed. Appl. Anim. Ethol. 10, 45-61. doi: 10.1016/0304-3762(83)90111-6

Caldji, C., Tannenbaum, B., Sharma, S., Francis, D., Plotsky, P. M., and Meaney, M. J. (1998). Maternal care during infancy regulates the development of neural systems mediating the expression of fearfulness in the rat. Proc. Natl. Acad. Sci. U.S.A. 95, 5335-5340. doi: 10.1073/pnas.95.9.5335

Champagne, F. A., Francis, D. D., Mar, A., and Meaney, M. J. (2003). Variations in maternal care in the rat as a mediating influence for the effects of environment on development. Physiol. Behav. 79, 359-371. doi: 10.1016/S0031-9384(03)00149-5

Champagne, F. A., and Meaney, M. J. (2006). Stress during gestation alters postpartum maternal care and the development of the offspring in a rodent model. Biol. Psychiatry 59, 1227-1235. doi: 10.1016/j.biopsych.2005.10.016

Clayton, N. S., and Krebs, J. R. (1994). Hippocampal growth and attrition in birds affected by experience. Proc. Natl. Acad. Sci. U.S.A. 91, 7410-7414. doi: $10.1073 /$ pnas. 91.16 .7410

Coppola, C. L., Grandin, T., and Enns, R. M. (2006). Human interaction and cortisol: can human contact reduce stress for shelter dogs? Physiol. Behav. 87, 537-541. doi: 10.1016/j.physbeh.2005.12.001

Czerwinski, V. H., McArthur, M., Smith, B., Hynd, P., and Hazel, S. (2016a). Selection of breeding stock among Australian purebred dog breeders, with particular emphasis on the dam. Animals 6, 1-18. doi: 10.3390/ani 6110075

Czerwinski, V. H., Smith, B. P., Hynd, P. I., and Hazel, S. J. (2016b). The influence of maternal care on stress-related behaviors in domestic dogs: responsibility of the authors and do not necessarily reflect the views of the National Science Foundation.

\section{ACKNOWLEDGMENTS}

We thank S. Bartner, S. Frommer, N. Gay, A. Gersick, R. Schwartz, A. Seely, and M. Torres for assistance with data collection, dog testing, and coding. We also thank S. Hasan for his enormous help with Datavyu export code. J. Parker and W. Lundskow from Salimetrics and K. Henning from the Institute for Interdisciplinary Salivary Bioscience Research at Arizona State University were very helpful in advising on and coordinating saliva collection and analysis. We're especially grateful to Dr. Dolores Holle for coordinating our work at The Seeing Eye, as well as the leadership team of The Seeing Eye, Breeding Station Manager Maria Hevner, and the breeding station staff for giving us access to the breeding station and permitting us to work with their mothers and litters.

what can we learn from the rodent literature? J. Vet. Behav. 14, 52-59. doi: 10.1016/j.jveb.2016.05.003

DatavyuTeam (2014). Datavyu: A Video Coding Tool. New York, NY: Databrary Project, New York University.

DeVore, E. R., and Ginsburg, K. R. (2005). The protective effects of good parenting on adolescents. Curr. Opin. Pediatr. 17, 460-465. doi: 10.1097/01.mop.0000170514.27649.c9

Dinno, A. (2012). Paran: Horn's Test of Principal Components/Factors. R package version 1.5.1. Available online at: http://www.alexisdinno.com/stata/paran. html

Dreschel, N. A., and Granger, D. A. (2009). Methods of collection for salivary cortisol measurement in dogs. Horm. Behav. 55, 163-168. doi: 10.1016/j.yhbeh.2008.09.010

Dwyer, C., and Lawrence, A. (2000). Maternal behaviour in domestic sheep (Ovis Aries): constancy and change with maternal experience. Behaviour 137, 1391-1413. doi: 10.1163/156853900501999

Fairbanks, L. A. (1996). Individual differences in maternal style: causes and consequences for mothers and offspring. Adv. Study Behav. 25, 579-611. doi: 10.1016/S0065-3454(08)60343-5

Fält, L., and Wilsson, E. (1979). The effect of maternal deprivation between 6 and 10 weeks of age upon the behaviour of alsatian puppies. Appl. Anim. Ethol. 5, 299. doi: 10.1016/0304-3762(79)90077-4

Field, A., Miles, J., and Field, Z. (2012). Discovering Statistics Using, R. London: SAGE Publications Ltd.

Fish, E. W., Shahrokh, D., Bagot, R., Caldji, C., Bredy, T., Szyf, M., et al. (2004). Epigenetic programming of stress responses through variations in maternal care. Ann. N.Y. Acad. Sci. 1036, 167-180. doi: 10.1196/annals. 1330.011

Foyer, P., Wilsson, E., and Jensen, P. (2016). Levels of maternal care in dogs affect adult offspring temperament. Sci. Rep. 6, 1-8. doi: 10.1038/srep19253

Foyer, P., Wilsson, E., Wright, D., and Jensen, P. (2013). Early experiences modulate stress coping in a population of German shepherd dogs. Appl. Anim. Behav. Sci. 79-87. doi: 10.1016/j.applanim.2013. 03.013

Francis, D., Diorio, J., Liu, D., and Meaney, M. J. (1999). Nongenomic transmission across generations of maternal behavior and stress responses in the rat. Science 286, 1155-1158. doi: 10.1126/science.286.5442.1155

Guardini, G., Bowen, J., Raviglione, S., Farina, R., and Gazzano, A. (2015). Maternal behaviour in domestic dogs: a comparison between primiparous and multiparous dogs. Dog Behav. 1, 23-33. doi: 10.4454/db.v1i1.4

Guardini, G., Mariti, C., Bowen, J., Fatjó, J., Ruzzante, S., Martorell, A., et al. (2016) Influence of morning maternal care on the behavioural responses of 8-week-old 
Beagle puppies to new environmental and social stimuli. Appl. Anim. Behav. Sci. 181, 137-144. doi: 10.1016/j.applanim.2016.05.006

Halekoh, U., Højsgaard, S., and Yan, J. (2006). The R package geepack for generalized estimating equations. J. Stat. Softw. 15, 1-11. doi: $10.18637 /$ jss.v015.i02

Hoeve, M., Dubas, J. S., Eichelsheim, V. I., Van der Laan, P. H., Smeenk, W., and Gerris, J. R. (2009). The relationship between parenting and delinquency: a meta-analysis. J. Abnorm. Child Psychol. 37, 749-775. doi: $10.1007 /$ s10802-009-9310-8

Horn, J. L. (1965). A rationale and test for the number of factors in factor analysis. Psychometrika 30, 179-185. doi: 10.1007/BF02289447

Johnston, B. (1990). The Skilful Mind of the Guide Dog: Towards a Cognitive and Holistic Model of Training. Windsor, ON: GDBA.

Kobelt, A. J., Hemsworth, P. H., Barnett, J. L., and Butler, K. L. (2003). Sources of sampling variation in saliva cortisol in dogs. Res. Vet. Sci. 75, 157-161. doi: 10.1016/S0034-5288(03)00080-8

Kosten, T. A., Lee, H. J., and Kim, J. J. (2006). Early life stress impairs fear conditioning in adult male and female rats. Brain Res. 1087, 142-150. doi: 10.1016/j.brainres.2006.03.009

Leroy, G., Verrier, E., Wisner-Bourgeois, C., and Rognon, X. (2007). Breeding goals and breeding practices of French dog breeders: results from a large survey. Revue Méd. Vét. 158, 496-503.

Liang, K.-Y., and Zeger, S. L. (1993). Regression analysis for correlated data. Annu. Rev. Public Health 14, 43-68. doi: 10.1146/annurev.pu.14.050193.000355

Liu, D., Diorio, J., Day, J. C., Francis, D. D., and Meaney, M. J. (2000). Maternal care, hippocampal synaptogenesis and cognitive development in rats. Nat. Neurosci. 3, 799-806. doi: 10.1038/77702

Liu, D., Diorio, J., Tannenbaum, B., Caldji, C., Francis, D., Freedman, A., et al. (1997). Maternal care, hippocampal glucocorticoid receptors, and hypothalamic-pituitary-adrenal responses to stress. Science 277, 1659-1662. doi: $10.1126 /$ science.277.5332.1659

Lopez-Duran, N. L., Hajal, N. J., Olson, S. L., Felt, B. T., and Vazquez, D. M. (2009). Individual differences in cortisol responses to fear and frustration during middle childhood. J. Exp. Child Psychol. 103, 285-295. doi: 10.1016/j.jecp.2009.03.008

Maestripieri, D. (1993). Maternal anxiety in rhesus macaques (Macaca mulatta). Ethology 95, 19-31. doi: 10.1111/j.1439-0310.1993.tb00453.x

Maestripieri, D., McCormack, K., Lindell, S. G., Higley, J. D., and Sanchez, M. M. (2006). Influence of parenting style on the offspring's behaviour and CSF monoamine metabolite levels in crossfostered and noncrossfostered female rhesus macaques. Behav. Brain Res. 175, 90-95. doi: 10.1016/j.bbr.2006.08.002

Meaney, M. J. (2001). Maternal care, gene expression, and the transmission of individual differences in stress reactivity across generations. Neuroscience 24, 1161-1192. doi: 10.1146/annurev.neuro.24.1.1161

Morey, D. F. (1994). The early evolution of the domestic dog. Am. Sci. 82, 336-347.

Myers, M. M., Brunelli, S. A., Shair, H. N., Squire, J. M., and Hofer, M. A. (1989a). Relationships between maternal behavior of SHR and WKY dams and adult blood pressures of cross-fostered F1 pups. Dev. Psychobiol. 22, 55-67. doi: 10.1002/dev.420220105

Myers, M. M., Brunelli, S. A., Squire, J. M., Shindeldecker, R. D., and Hofer, M. A. (1989b). Maternal behavior of SHR rats and its relationship to offspring blood pressures. Dev. Psychobiol. 22, 29-53. doi: 10.1002/dev.420220104

Parker, K. J., and Maestripieri, D. (2011). Identifying key features of early stressful experiences that produce stress vulnerability and resilience in primates. Neurosci. Biobehav. Rev. 35, 1466-1483. doi: 10.1016/j.neubiorev.2010.09.003

Pierantoni, L., Albertini, M., and Pirrone, F. (2011). Prevalence of owner-reported behaviours in dogs separated from the litter at two different ages. Vet. Rec. Eng. Ed. 169, 468. doi: 10.1136/vr.d4967
Pierantoni, L., and Verga, M. (2007). Behavioral consequences of premature maternal separation and lack of stimulation during the socialization period in dogs. J. Vet. Behav. 2, 84-85. doi: 10.1016/j.jveb.2007.04.009

Pittet, F., Houdelier, C., de Margerie, E., Le Bot, O., Richard-Yris, M.-A., and Lumineau, S. (2014). Maternal styles in a precocial bird. Anim. Behav. 87, 31-37. doi: 10.1016/j.anbehav.2013.10.025

Priestnall, R. (1972). Effects of litter size on the behaviour of lactating female mice (Mus musculus). Anim. Behav. 20, 386-394. doi: 10.1016/S0003-3472(72)80063-0

Pryce, C. R., Dettling, A., Spengler, M., Spaete, C., and Feldon, J. (2004). Evidence for altered monoamine activity and emotional and cognitive disturbance in marmoset monkeys exposed to early life stress. Ann. N.Y. Acad. Sci. 1032, 245-249. doi: 10.1196/annals.1314.030

Rao, H., Betancourt, L., Giannetta, J. M., Brodsky, N. L., Korczykowski, M., Avants, B. B., et al. (2010). Early parental care is important for hippocampal maturation: evidence from brain morphology in humans. Neuroimage 49, 1144-1150. doi: 10.1016/j.neuroimage.2009.07.003

R Development Core Team (2016). R: A Language and Environment for Statistical Computing. Vienna: R Foundation for Statistical Computing.

Repetti, R. L., Taylor, S. E., and Seeman, T. E. (2002). Risky families: family social environments and the mental and physical health of offspring. Psychol. Bull. 128, 330-366. doi: 10.1037/0033-2909.128.2.330

Ruscio, J., and Roche, B. (2012). Determining the number of factors to retain in an exploratory factor analysis using comparison data of known factorial structure. Psychol. Assess. 24, 282-292. doi: 10.1037/a0025697

Serpell, J. A., Duffy, D. L., and Jagoe, J. A. (2016). “Becoming a dog: early experience and the development of behavior," in The Domestic Dog, ed J. A. Serpell (New York, NY: Cambridge University Press), 93-117.

Seyfarth, R. M., Silk, J. B., and Cheney, D. L. (2012). Variation in personality and fitness in wild female baboons. Proc. Natl. Acad. Sci. U.S.A. 109, 16980-16985. doi: 10.1073/pnas.1210780109

Tiira, K., and Lohi, H. (2015). Early life experiences and exercise associate with canine anxieties. PLoS ONE 10:e0141907. doi: 10.1371/journal.pone.0141907

van der Waaij, E. H., Wilsson, E., and Strandberg, E. (2008). Genetic analysis of results of a Swedish behavior test on German Shepherd Dogs and Labrador Retrievers. J. Anim. Sci. 86, 2853-2861. doi: 10.2527/jas.2007-0616

van IJzendoorn, M. H., Moran, G., Belsky, J., Pederson, D., BakermansKranenburg, M. J., and Kneppers, K. (2000). The similarity of siblings' attachments to their mother. Child Dev. 71, 1086-1098. doi: 10.1111/1467-8624.00211

Wilsson, E., and Sundgren, P.-E. (1998). Effects of weight, litter size and parity of mother on the behaviour of the puppy and the adult dog. Appl. Anim. Behav. Sci. 56, 245-254. doi: 10.1016/S0168-1591(97)00094-4

Conflict of Interest Statement: The authors declare that the research was conducted in the absence of any commercial or financial relationships that could be construed as a potential conflict of interest.

The reviewer FR and the handling Editor declared their shared affiliation, and the handling Editor states that the process nevertheless met the standards of a fair and objective review.

Copyright (C) 2017 Bray, Sammel, Cheney, Serpell and Seyfarth. This is an open-access article distributed under the terms of the Creative Commons Attribution License (CC $B Y)$. The use, distribution or reproduction in other forums is permitted, provided the original author(s) or licensor are credited and that the original publication in this journal is cited, in accordance with accepted academic practice. No use, distribution or reproduction is permitted which does not comply with these terms. 